\title{
Successive Minima, Intrinsic Volumes, and Lattice Determinants
}

\author{
U. Schnell \\ Mathematisches Institut, Universität Siegen, \\ D-57068 Siegen, Germany \\ schnell@mathematik.uni-siegen.d400.de
}

Communicated by Jörg M. Wills

\begin{abstract}
In Euclidean $d$-space $E^{d}$ we prove a lattice-point inequality for arbitrary lattices and for the intrinsic volumes $V_{i}$ (i.e., normalized quermassintegrals) of convex bodies. The $V_{i}$ are not equi-affine invariant (except the volume), hence suitable functionals of the lattice have to be introduced. The result generalizes an earlier result of Henk for the integer lattice $\mathbb{Z}^{d}$.
\end{abstract}

\section{Introduction and Results}

In the following let $E^{d}, d \geq 2$, denote the Euclidean $d$-space and let $\mathscr{L}^{d}$ denote the set of lattices $L \subset E^{d}$ with $\operatorname{det}(L) \neq 0$. Further, let $\mathscr{K}^{d}$ denote the set of convex bodies $K \subset E^{d}$ and let $\mathscr{K}_{0}^{d}$ denote the subset of 0 -symmetric convex bodies. For $K \in \mathscr{K}^{d}$ let $V_{i}(K), i=0, \ldots, d$, be its intrinsic volumes or normalized quermassintegrals (see [7]). In particular, $V_{d}(K)=V(K)$ is the volume and $V_{d-1}(K)=\frac{1}{2} F(K)$ is the half-surface area of $K$. For $K \in \mathscr{K}_{0}^{d}$ and $L \in \mathscr{L}^{d}$ let $\lambda_{i}(K, L)$ denote the $i$ th successive minimum of $K$ with respect to the lattice $L$. For the special case $K=B^{d}$ we have the successive minima $\lambda_{i}(L)=\lambda_{i}\left(B^{d}, L\right)$ of the lattice $L$ (see [6]).

For centrally symmetric $K \in \mathscr{K}_{0}^{d t}$ Henk [5] proved

$$
\lambda_{i+1}\left(K, \mathbb{Z}^{d}\right) \cdot \cdots \cdot \lambda_{d}\left(K, \mathbb{Z}^{d}\right) V_{d}(K) \leq 2^{d-i} V_{i}(K), \quad i=1, \ldots, d-1,
$$

which for $i=0$ is Minkowski's second theorem (see p. 59 of [2]).

Clearly, a generalization of (1) to arbitrary lattices is desirable. The problem is that the proof of (1) uses special properties of $\mathbb{Z}^{d}$, and that the $V_{i}$ (except $V_{d}$ ) are not equi-affine invariant. The basic idea to overcome these difficulties is to introduce functionals of $L$, which correspond to the $V_{i}$ as, e.g., the minimal determinants (see [14]),

$$
D_{i}(L)=\min \left\{\operatorname{det}\left(L_{i}\right): L_{i} \text { is an } i \text {-dimensional sublattice of } L\right\}, \quad i=1, \ldots, d,
$$


and $D_{0}(L)=1$. Obviously $D_{i}\left(\mathbb{Z}^{d}\right)=1, i=0, \ldots, d$, and $D_{d}(L)=\operatorname{det}(L)$. With $D_{i}$ and the last successive minimum $\lambda_{d}$ of the lattice, generalizations of a lattice-point inequality for convex bodies by Bokowski et al. [1] and of an isoperimetric inequality for lattice periodic sets by Hadwiger [4] for the integer lattice $\mathbb{Z}^{d}$ to arbitrary lattices have been given (see [8]-[11]).

Further, the following generalization of (1) is conjectured.

Conjecture. Let $K \in \mathscr{K}_{0}^{d}$ and $L \in \mathscr{L}^{d}$. Then

$$
\lambda_{i+1}(K, L) \cdot \cdots \cdot \lambda_{d}(K, L) \frac{V_{d}(K)}{D_{d}(L)}<2^{d-i} \frac{V_{i}(K)}{D_{i}(L)}, \quad i=1, \ldots, d-1 .
$$

A first result of this type is given implicitly by Wills [13]. He proved that (2) is true if the factor $i$ ! is added to the right-hand side.

In Section 2 we prove that the Conjecture is best possible for each lattice.

A proof of the Conjecture seems to be hard. It is the purpose of this paper to introduce some related lattice functionals instead of $D_{i}$ and to prove tight inequalities related to (1) for arbitrary $L \in \mathscr{L}^{d}$. These functionals are

$$
C_{i}(L)=\max _{L_{d-i}} \min _{L_{d-i} \cap L_{i}=\{0\}} \operatorname{det}\left(L_{i}\right), \quad i=1, \ldots, d,
$$

where $L_{i}$ and $L_{d-i}$ are $i$ - and $(d-i)$-dimensional sublattices of $L$, respectively, but not necessarily $L_{i}+L_{d-i}=L$. Further, let $C_{0}(L)=1$. Obviously $C_{d}(L)=\operatorname{det}(L)$ and $C_{i}\left(\mathbb{Z}^{d}\right)=1, i=0, \ldots, d$. These and other properties of $C_{i}$ are collected in the following proposition.

\section{Proposition.}

(a) The $C_{i}$ are invariant under rigid motion and homogeneous of degree $i$.

(b) Let $k_{1} \leq \cdots \leq k_{d}$ and $i=1, \ldots, d$. Then

$$
C_{i}\left(\operatorname{diag}\left(k_{1}, \ldots, k_{d}\right) \mathbb{Z}^{d}\right)=k_{d-i+1} \cdot \cdots \cdot k_{d} .
$$

(c) $C_{d}(L)=D_{d}(L)=\operatorname{det}(L)$.

(d) The $C_{i}$ exist and $C_{i}(L) \leq \lambda_{d-i+1}(L) \cdot \cdots \cdot \lambda_{d}(L), i=1, \ldots, d$.

(e) $C_{1}(L)=\lambda_{d}(L)$.

(f) $C_{i}(L) \geq D_{i}(L), i=0, \ldots, d$.

(g) $D_{i+j}(L) \leq D_{i}(L) C_{j}(L), i=0, \ldots, d ; j=0, \ldots, d-i$.

(h) For each inequality there is an $L$ with strict inequality.

Our main result is now:

Theorem 1. Let $K \in \mathscr{K}_{0}^{d}$ and $L \in \mathscr{L}^{d}$. Then

$$
\lambda_{i+1}(K, L) \cdot \cdots \cdot \lambda_{d}(K, L) V_{d}(K) \leq 2^{d-i} C_{d-i}(L) V_{i}(K), \quad i=0, \ldots, d-1 .
$$

This inequality is tight, i.e., $C_{i}(L)$ cannot be replaced by $C_{i}(L)-\varepsilon$. From (b) in the Proposition it follows that Theorem 1 generalizes (1) and from $(\mathrm{g})$ and $(\mathrm{h})$ in the 
Proposition it follows that Theorem 1 is weaker than the Conjecture. From (d) and (h) of the Proposition it follows that Theorem 1 is an improvement of (see [11])

$$
\begin{aligned}
& \lambda_{i+1}(K, L) \cdot \cdots \cdot \lambda_{d}(K, L) V_{d}(K) \\
& \quad \leq 2^{d-i} \lambda_{i+1}(L) \cdot \cdots \cdot \lambda_{d}(L) V_{i}(K), \quad i=0, \ldots, d-1 .
\end{aligned}
$$

Further, we can give the following geometric interpretation of the relation between $\lambda_{i}$ and $V_{i}$ :

Corollary 1. Let $K \in \mathscr{K}_{0}^{d}$ and $L \in \mathscr{L}^{d}$. Then (with $\lambda_{i}=\lambda_{i}(K, L)$ )

$$
V_{d}\left(\frac{1}{2} \lambda_{i+1} K\right) \leq C_{d-i}(L) V_{i}\left(\frac{1}{2} \lambda_{i+1} K\right), \quad i=0, \ldots, d-1 .
$$

For $i=0$ Corollary 1 is Minkowski's main theorem in Geometry of Numbers.

In Section 3 we give some basic properties of $C_{i}$. In particular we prove the Proposition and the following theorem.

Theorem 2. Let $L \in \mathscr{L}^{d}$. Then

$$
C_{i}(L)=C_{d}(L) C_{d-i}\left(L^{*}\right), \quad i=0, \ldots, d,
$$

where $L^{*}$ is the dual lattice of $L$.

\section{Tightness of the Conjecture}

Now we give a sequence of convex bodies such that the defect in the Conjecture tends to zero. For $i=0$ the Conjecture is the second theorem of Minkowski, which is tight for each lattice (e.g., for the DV- or Voronoi-cell (see, e.g., [2]) of the lattice, equality holds). Here we consider the case $1 \leq i \leq d-1$. Let $L_{i}$ be an $i$-dimensional sublattice of $L$ with $\operatorname{det}\left(L_{i}\right)=D_{i}(L)$ and let $E_{i}=\operatorname{lin}\left(L_{i}\right)$. Further, let $K_{0} \subset E_{i}$ temporarily be an arbitrary convex body and let $Z$ be the DV-cell of the $\left(d-i\right.$ )-dimensional lattice $L / E_{i}{ }^{\perp}$ (where / denotes the orthogonal projection), then it follows from Lemma 1 in [8] that

$$
V_{d-i}(Z)=\operatorname{det}\left(L / E_{i}{ }^{\perp}\right)=\frac{\operatorname{det}(L)}{\operatorname{det}\left(L_{i}\right)}=\frac{D_{d}(L)}{D_{i}(L)},
$$

where $V_{j}$ denotes the $j$-dimensional volume. In the following let $K:=K_{0}+Z$. We have

$$
\lambda_{i+j}(K, L) \geq \lambda_{j}\left(K / E_{i}^{\perp}, L / E_{i}^{\perp}\right)=\lambda_{j}\left(Z, L / E_{i}^{\perp}\right)=2
$$

Further,

$$
V_{d}(K)=V_{i}\left(K_{0}\right) V_{d-i}(Z)=\frac{V_{i}\left(K_{0}\right) D_{d}(L)}{D_{i}(L)}
$$


Hence it follows that

$$
\frac{2^{d-i} V_{i}(K) D_{d}(L)}{D_{i}(L) \lambda_{i+1}(K, L) \cdot \cdots \cdot \lambda_{d}(K, L) V_{d}(K)} \leq \frac{V_{i}(K) D_{d}(L)}{D_{i}(L) V_{d}(K)}=\frac{V_{i}\left(K_{0}+Z\right)}{V_{i}\left(K_{0}\right)} .
$$

So it suffices to give a sequence of $K_{0}$ such that $V_{i}\left(K_{0}+Z\right) / V_{i}\left(K_{0}\right)$ tends to one.

If we write the formula for quermassintegrals of a sum of convex bodies lying in complementary subspaces (see p. 215 of [3]) in terms of the intrinsic volumes and apply it to $K_{0}+Z$ we obtain

$$
V_{i}\left(K_{0}+Z\right)=\sum_{\nu=0}^{d-i} V_{i-\nu}\left(K_{0}\right) V_{\nu}(Z)=V_{i}\left(K_{0}\right)+\sum_{\nu=1}^{d-i} V_{i-\nu}\left(K_{0}\right) V_{\nu}(Z)
$$

With $R:=\max _{1 \leq \nu \leq d-i} V_{\nu}(Z)$ it follows that $V_{i}\left(K_{0}+Z\right) \leq V_{i}\left(K_{0}\right)+$ $R \sum_{\nu=0}^{i-1} V_{\nu}\left(K_{0}\right)$.

Now let $K_{0}:=r B^{i}$ be the ball with radius $r>0$, then it follows, with $V_{i}\left(B^{d}\right)=$ $\left(\begin{array}{c}d \\ i\end{array}\right) \kappa_{d} / \kappa_{d-i}$ (where $\kappa_{j}$ denotes the volume of the $j$-dimensional unit ball), that

$$
\begin{aligned}
\frac{V_{i}\left(K_{0}+Z\right)}{V_{i}\left(K_{0}\right)} & \leq 1+R \sum_{\nu=0}^{i-1} \frac{V_{\nu}\left(K_{0}\right)}{V_{i}\left(K_{0}\right)} \leq 1+R \sum_{\nu=0}^{i-1} \frac{r^{\nu}\left(\begin{array}{l}
i \\
\nu
\end{array}\right) \frac{\kappa_{i}}{\kappa_{i-\nu}}}{r^{i}\left(\begin{array}{l}
i \\
i
\end{array}\right) \frac{\kappa_{i}}{\kappa_{0}}} \\
& =1+R \sum_{\nu=0}^{i-1} r^{\nu-i} \frac{\left(\begin{array}{l}
i \\
\nu
\end{array}\right)}{\kappa_{i-\nu}} \rightarrow 1 \quad(r \rightarrow \infty)
\end{aligned}
$$

\section{Properties of $C_{i}$}

To give a slightly different definition of $C_{i}$ we need the following lemma, which is a straightforward application of the dimension formula for submodules (see p. 120 of [12]) and for linear subspaces, respectively.

Lemma 1. Let $L_{i}$ and $L_{d-i}$ be $i$ - and $(d-i)$-dimensional sublattices of a lattice $L \in \mathscr{L}^{d}$, respectively. Then

$$
L_{d-i} \cap L_{i}=\{0\} \quad \Leftrightarrow \quad \operatorname{lin}\left(L_{d-i}\right) \cap \operatorname{lin}\left(L_{i}\right)=\{0\},
$$

where $\operatorname{lin}(M)$ is the linear hull of the set $M$.

The following lemma shows that we can maximize over arbitrary subspaces instead of sublattices in the definition of $C_{i}$.

Lemma 2. Let $L \in \mathscr{L}^{d}$. Then

$$
C_{i}(L)=\max _{E_{d-\iota}} \min _{E_{d-i} \cap L_{i}=\{0\}} \operatorname{det}\left(L_{i}\right),
$$

where $E_{d-i}$ is an arbitrary $(d-i)$-dimensional subspace of $E^{d}$. 
Proof. Let $E_{d-i}$ be an arbitrary $(d-i)$-dimensional subspace of $E^{d}$ and let $L_{r}:=L \cap E_{d-i}$ be an $r$-dimensional sublattice of $L(0 \leq r \leq d-i)$. Then we can complete $L_{r}$ to a $(d-i)$-dimensional sublattice $L_{d-i}$ of $L$, such that $L_{r} \subset L_{d-i}$. If $L_{d-i} \cap L_{i}=\{0\}$, then $L_{r} \cap L_{i}=\{0\}$ and so $E_{d-i} \cap L_{i}=E_{d-i} \cap L \cap L_{i}=$ $L_{r} \cap L_{i}=\{0\}$. Consequently, we have

$$
\min _{L_{d-i} \cap L_{i}=\{0\}} \operatorname{det}\left(L_{i}\right) \geq \min _{E_{d-i} \cap L_{i}=\{0\}} \operatorname{det}\left(L_{i}\right)
$$

which yields " $\geq$." The reverse inequality follows from Lemma 1 .

Proof of the Proposition. (a), (b), and (c) are clear, (f) follows from (3).

To prove (d) let $u_{j} \in \lambda_{j}\left(B^{d}, L\right) B^{d} \cap L, j=1, \ldots, d$, be $d$ linearly independent lattice points. Then $\left|u_{j}\right|=\lambda_{j}(L)$ and $U=\left\{u_{1}, \ldots, u_{d}\right\}$ forms a basis of $E^{d}$. Let $L_{d-i}$ be a $(d-i)$-dimensional sublattice of $L$ and let $A=\left\{a_{1}, \ldots, a_{d-i}\right\}$ be a basis of $L_{d-i}$. Let $U_{1} \subset U$ be a maximal subset of $U$ with $A \cup U_{1}$ linearly independent. Then $u \in \operatorname{lin}\left(A \cup U_{1}\right)$ for all $u \in U$ and so $A \cup U_{1}$ forms a basis of $E^{d}$. Hence we have $\left|U_{1}\right|=i$ and the $i$-dimensional lattice $L_{i}$ spanned by $U_{1}$ has the property $\operatorname{dim}\left(L_{d-i}+L_{i}\right)=d$ and so we can conclude, as in the proof of Lemma 1 , that $L_{d-i} \cap L_{i}=\{0\}$. Now (d) follows from

$$
\operatorname{det}\left(L_{i}\right) \leq \prod_{u \in U_{1}}|u| \leq \lambda_{d-i+1}(L) \cdot \cdots \cdot \lambda_{d}(L) .
$$

To prove (e) it suffices to prove $C_{1} \geq \lambda_{d}$ (we omit the $L$ ). If $\lambda_{d}>C_{1}$, then there is an $r \geq 1$ such that $\lambda_{r+1}>C_{1} \geq \lambda_{r}$, since $C_{1} \geq D_{1}=\lambda_{1}$. Let $u_{1}, \ldots, u_{r} \in L$ with $\left|u_{i}\right|=\lambda_{i}, i=1, \ldots, r$, and let $L_{r}$ be the $r$-dimensional sublattice spanned by $u_{1}, \ldots, u_{r}$. Then we can complete $L_{r}$ to a $(d-1)$-dimensional sublattice $L_{d-1}$ and by the definition of $C_{1}$ a lattice vector $u_{r+1} \notin L_{d-1}$ with $\left|u_{r+1}\right| \leq C_{1}<\lambda_{r+1}$ exists. Since $u_{1}, \ldots, u_{r+1}$ are linearly independent, this is a contradiction to the definition of $\lambda_{r+1}$.

In (g) the cases $i=0, i=d$, and $j=0$ are clear. For $1 \leq i \leq d-1$ and $1 \leq j \leq d-i$ let $L_{i}$ be an $i$-dimensional sublattice of $L$ with $\operatorname{det}\left(L_{i}\right)=D_{i}(L)$. We can complete $L_{i}$ to a $(d-j)$-dimensional sublattice $L_{d-j}$ with $E_{d-j}:=\operatorname{lin}\left(L_{d-j}\right)$. Consider the lattice $L_{j}$ with $L_{j} \cap E_{d-j}=\{0\}$ and minimal determinant, then

$$
C_{j}(L) \geq \operatorname{det}\left(L_{j}\right)
$$

Let $P_{1}$ and $P_{2}$ be the fundamental epipeds to $L_{i}$ and $L_{j}$, respectively. Then $P=P_{1}+P_{2}$ is a fundamental epiped to the $(i+j)$-dimensional sublattice $L_{i+j}=$ $L_{i}+L_{j}$. With the principle of Cavalieri and (5) it follows that

$$
\begin{aligned}
V_{i+j}(P) & =V_{i}\left(P_{1}\right) V_{j}\left(P_{2} / E_{d-j}^{\perp}\right) \leq V_{i}\left(P_{1}\right) V_{j}\left(P_{2}\right) \\
& =\operatorname{det}\left(L_{i}\right) \operatorname{det}\left(L_{j}\right) \leq D_{i}(L) C_{j}(L) .
\end{aligned}
$$

Now (g) follows from $D_{i+j}(L) \leq \operatorname{det}\left(L_{i+j}\right)=V_{i+j}(P)$. 
Now we prove (h). For (f) choose the lattice with basis $\left\{e_{1}, 2 e_{2}, \ldots, d e_{d}\right\}$. Then $D_{i}=i !$ and $C_{i}=d ! /(d-i) !$ and so $C_{i} / D_{i}=\left(\begin{array}{c}d \\ i\end{array}\right)>1$, for $i=1, \ldots, d-1$. For (d) and (g) we choose a lattice $L$, such that $v \cdot w \neq 0$, for all $v, w \in L \backslash\{0\}$ (e.g., the lattice with basis $(1,0, \ldots, 0)^{t},(\pi, \pi, 0, \ldots, 0)^{t},\left(\pi^{2}, \pi^{2}, \pi^{2}, 0, \ldots, 0\right)^{t}$, $\ldots,\left(\pi^{d-1}, \ldots, \pi^{d-1}\right)^{t}$ has this property since $\pi$ is transcendental). Then we have, in (4) and (6) (in (4) only for $i>1$ ) in the proofs of (d) and (g), strict inequality.

Proof of Theorem 2. For $i=0$ and $i=d$ the assertion follows from $C_{0}(L)=1$ and $C_{d}(L)=\operatorname{det}(L)$. For $1 \leq i \leq d-1$ it suffices to prove $C_{i}(L) \leq C_{d-i}\left(L^{*}\right) \operatorname{det}(L)$, because we can apply this to $L^{*}$ and $d-i$ instead of $i$ and obtain the reverse inequality.

Let $L_{d-i}$ be a $(d-i)$-dimensional sublattice, such that

$$
C_{i}(L)=\min _{L_{d-i} \cap \Lambda_{i}=\{0\}} \operatorname{det}\left(\Lambda_{i}\right)
$$

and let $E_{d-i}=\operatorname{lin}\left(L_{d-i}\right)$, then $E_{d-i}^{\perp}$ is an $i$-dimensional subspace, which is spanned by a sublattice of $L^{*}$ (see [8]). Let $\tilde{L}_{d-i}$ be a $(d-i)$-dimensional sublattice of $L^{*}$ with $\tilde{L}_{d-i} \cap E_{d-i}^{\perp}=\{0\}$ and $\operatorname{det}\left(\tilde{L}_{d-i}\right)$ minimal, then

$$
C_{d-i}\left(L^{*}\right) \geq \operatorname{det}\left(\tilde{L}_{d-i}\right)
$$

We can assume that $\tilde{L}_{d-i}$ is primitive in $L^{*}$, since from Lemma 1 it follows that $\operatorname{lin}\left(\tilde{L}_{d-i}\right) \cap E_{d-i}^{\perp}=\{0\}$, and otherwise the lattice $L^{*} \cap \operatorname{lin}\left(\tilde{L}_{d-i}\right)$ would be a "better" lattice. Now let $L_{i}:=L \cap\left(\operatorname{lin}\left(\tilde{L}_{d-i}\right)\right)^{\perp}$, then $L_{i}$ is an $i$-dimensional sublattice of $L$ with $L_{i} \cap E_{d-i}=\{0\}$.

Let $x \in L_{i} \cap E_{d-i}$. As in the proof of Lemma 1, we can show that $\operatorname{lin}\left(\tilde{L}_{d-i}\right)+$ $E_{d-i}^{\perp}=E^{d}$, i.e., we can represent each $y \in E^{d}$ as $y=u+v$, where $u \in \operatorname{lin}\left(\tilde{L}_{d-i}\right)$ and $v \in E_{d-i}^{\perp}$. Then it follows that $x \cdot y=x \cdot u+x \cdot v=0+0=0$, since $x \in L_{i} \subset$ $\left(\operatorname{lin}\left(\tilde{L}_{d-i}\right)\right)^{\perp}$ and $x \in E_{d-i}=\left(E_{d-i}^{\perp}\right)^{\perp}$. Consequently, $x=0$.

Hence we have

$$
\operatorname{det}\left(L_{i}\right) \geq \min _{L_{d-i} \cap \Lambda_{i}=\{0\}} \operatorname{det}\left(\Lambda_{i}\right)=C_{i}(L) .
$$

It further follows from [8], Theorem 1, and (8) that

$$
\operatorname{det}\left(\tilde{L}_{d-i}\right)=\operatorname{det}\left(L^{*}\right) \operatorname{det}\left(L_{i}\right)=\frac{\operatorname{det}\left(L_{i}\right)}{\operatorname{det}(L)} \geq \frac{C_{i}(L)}{\operatorname{det}(L)} .
$$

Finally it follows from (7) and (9) that

$$
C_{d-i}\left(L^{*}\right) \geq \operatorname{det}\left(\tilde{L}_{d-i}\right) \geq \frac{C_{i}(L)}{\operatorname{det}(L)},
$$

and Theorem 2 is proved.

\section{Proof of Theorem 1}

For $i=0$ Theorem 1 is the second theorem of Minkowski, so it suffices to consider the case $1 \leq i \leq d-1$. For $j=1, \ldots, i$ let $y_{j} \in \lambda_{j}(K, L) K \cap L$ be $i$ linearly 
independent lattice points. Let $E_{i}=\operatorname{lin}\left\{y_{1}, \ldots, y_{i}\right\}$, then it follows by the definition of $C_{d-i}(L)$, that a $(d-i)$-dimensional sublattice $L_{d-i}$ of $L$ exists such that:

(1) $L_{d-i} \cap E_{i}=\{0\}$ (and, with Lemma 1 in Section 3, $\operatorname{lin}\left(L_{d-i}\right) \cap E_{i}=\{0\}$ ).

(2) $\operatorname{det}\left(L_{d-i}\right) \leq C_{d-i}(L)$.

Let $K_{d-i}:=K \cap \operatorname{lin}\left(L_{d-i}\right)$, then it follows, with the second fundamental theorem of Minkowski for $K_{d-i}$ and $L_{d-i}$, that

$$
\begin{gathered}
\lambda_{1}\left(K_{d-i}, L_{d-i}\right) \cdot \cdots \cdot \lambda_{d-i}\left(K_{d-i}, L_{d-i}\right) V_{d-i}\left(K_{d-i}\right) \\
\leq 2^{d-i} \operatorname{det}\left(L_{d-i}\right) \leq 2^{d-i} C_{d-i}(L) .
\end{gathered}
$$

By the choice of $L_{d-i}(\operatorname{see}(1)), \lambda_{j}\left(K_{d-i}, L_{d-i}\right) K$ contains $i+j$ linear-independent lattice points of $L$, such that

$$
\lambda_{i+j}(K, L) \leq \lambda_{j}\left(K_{d-i}, L_{d-i}\right), \quad j=1, \ldots, d-i,
$$

and so

$$
\lambda_{i+1}(K, L) \cdot \cdots \cdot \lambda_{d}(K, L) V_{d-i}\left(K_{d-i}\right) \leq 2^{d-i} C_{d-i}(L) .
$$

Now Theorem 1 follows from (10) and $V_{d}(K)<V_{i}(K) V_{d-i}\left(K_{d-i}\right)$ (see [5]).

\section{References}

1. J. Bokowski, H. Hadwiger, J. M. Wills, Eine Ungleichung zwischen Volumen, Oberfläche und Gitterpunktanzahl konvexer Körper im n-dimensionalen Raum, Math. Z. 127 (1972), 363-364.

2. P. M. Gruber, C. G. Lekkerkerker, Geometry of Numbers, North-Holland, Amsterdam, 1987.

3. H. Hadwiger, Vorlesungen über Inhalt, Oberfläche und Isoperimetrie, Springer-Verlag, Berlin, 1957.

4. H. Hadwiger, Gitterperiodische Punktmengen und Isoperimetrie, Monatsh. Math. 76 (1972), 410-418.

5. M. Henk, Inequalities between successive minima and intrinsic volumes of a convex body, Monatsh. Math. 110 (1990), 279-282.

6. J. C. Lagarias, H. W. Lenstra, Jr., C. P. Schnorr, Korkin-Zolotarev bases and successive minima of a lattice and its reciprocal lattice, Combinatorica 10(4) (1990), 333-348.

7. P. McMullen, Nonlinear angle-sum relations for polyhedral cones and polytopes, Math. Proc. Cambridge Philos. Soc. 78 (1975), 247-261.

8. U. Schnell, Minimal determinants and lattice inequalities, Bull. London Math. Soc. 24 (1992), 606-612.

9. U. Schnell, Lattice inequalities for convex bodies and arbitrary lattices, Monatsh. Math. 116 (1993), 331-337.

10. U. Schnell, J. M. Wills, Two isoperimetric inequalities with lattice constraints, Monatsh. Math. 112 (1991), 227-233.

11. U. Schnell, J. M. Wills, On successive minima and intrinsic volumes, Mathematika 40 (1993), 144-147.

12. B. L. Van der Waerden, Moderne Algebra, Part 2, Springer-Verlag, Berlin, 1931.

13. J. M. Wills, Minkowski's successive minima and the zeros of a convexity-function, Monatsh. Math. 109 (1990), 157-164.

14. J. M. Wills, Bounds for the lattice point enumerator, Geom. Dedicata 40 (1991), 237-244.

Received March 2, 1994, and in revised form July 11, 1994. 